\title{
Combining electromagnetic gyro-kinetic particle-in-cell simulations with collisions
}

\author{
Christoph Slaby*, Axel Könies, and Ralf Kleiber \\ Max-Planck-Institut für Plasmaphysik, \\ D-17491 Greifswald, Germany
}

(Dated: April 24, 2017)

\begin{abstract}
It has been an open question whether for electromagnetic gyro-kinetic particle-in-cell (PIC) simulations pitch-angle collisions and the recently introduced pullback transformation scheme [A. Mishchenko et al., Physics of Plasmas 21, 092110 (2014) and R. Kleiber et al., Physics of Plasmas 23, 032501 (2016)] are consistent.

This question is positively answered by comparing the PIC code EUTERPE with an approach based on an expansion of the perturbed distribution function in eigenfunctions of the pitchangle collision operator (Legendre polynomials) to solve the electromagnetic drift-kinetic equation with collisions in slab geometry.

It is shown how both approaches yield the same results for the frequency and damping rate of a kinetic Alfvén wave and how the perturbed distribution function is substantially changed by the presence of pitch-angle collisions.
\end{abstract}

Keywords: PIC simulation, pitch-angle collisions, kinetic Alfvén waves

PACS: 52.25Xz, 52.25Dg, 52.20Fs

\footnotetext{
* E-mail address: christoph.slaby@ipp.mpg.de
} 


\section{INTRODUCTION}

The numerical treatment of the gyro-kinetic equation including collisions and electromagnetic effects is, for instance, necessary for studying the interaction of Alfvén modes with fast particles. It has been shown analytically and numerically (see e.g. Refs. [2, 20]) that collisions have a particularly strong influence on the non-linear saturation mechanism of the mode by detrapping resonant particles. Also the multi-phase simulations addressing bursting phenomena for JT 60-U [4] and profile flattening effects for D III-D [27] rely on the proper treatment of collisions for the fast particles. Furthermore, trapped electrons can contribute to the damping of Alfvén waves as has been shown in Ref. [11]. (A more elaborate discussion can be found in Ref. [8].)

Most computational approaches for solving the gyro-kinetic equations either rely on a grid-based discretization (Eulerian codes) or use a particle discretization (particle-incell (PIC) codes). Additionally, the electromagnetic gyro-kinetic equations can either be stated in the so-called $v_{\|}$-formulation or $p_{\|}$-formulation, where the former uses the physical parallel velocity while the latter adopts the parallel canonical momentum $p_{\|}=$ $m v_{\|}+q A_{\|}$as an independent variable [5]. For PIC simulations, the $v_{\|}$-formulation had serious problems related to the partial time derivative of the perturbed parallel vector potential $\left(A_{\|}\right)$in the equations of motion [23]. Therefore, the $p_{\|}$-formulation [12] became standard despite its notorious cancellation problem (see e.g. Ref. [7]), caused by the appearance of a non-physical current in Ampère's law. This problem could be overcome by the adjustable control variate scheme [13]. A different approach, avoiding some problems of this method, is the recently proposed pullback transformation scheme (PTscheme) $[18,22]$. This algorithm, loosely speaking, works in $v_{\|}$. Thus, it was conjectured that it may provide an optimal framework for implementing a (pitch-angle) collision operator, which is naturally formulated in $v_{\|}$.

Here, the gyro-kinetic PIC code EUTERPE is used to investigate the compatibility of collisions with the PT-scheme (and additionally with the two-weight scheme developed in Ref. [24]) as well as its convergence properties. In the past, electrostatic calculations including collisions, neoclassical impurity transport studies as well as electromagnetic simulations without collisions were successfully performed with EUTERPE [9, 10, 17, 21].

In the present work, the PT-scheme is combined with a linearized electron pitch-angle collision operator. Since such a combination is reported for the first time, the main focus of this paper is on verification and not on validation. In order to concentrate on the essential properties of the numerical scheme, (drift-)kinetic Alfvén waves in a homogeneous slab are considered. Additionally, only electron pitch-angle collisions are included. Ion finite-Larmor-radius (FLR) effects are retained in the ion polarization density with a Padé approximation whereas the ion dynamics, electron FLR effects, and gyro-averaging of the fields over the gyro-ring are neglected. Gyro-averaging may contribute significantly to the damping from ion collisions when $k_{\perp} \rho_{\mathrm{i}} \gtrsim 1$ (with $k_{\perp}$ and $\rho_{\mathrm{i}}$ denoting the perpendicular wave vector of the mode and the ion gyro-radius, respectively). Notwithstanding the simplifications made for the collision operator, this problem is non-trivial and only few analytical results are available. Moreover, recent comparisons of frequencies and damping rates from analytical theory with direct mea- 
surements revealed striking discrepancies [26]. This additionally motivates the need for accurate algorithms to gain physical insight.

Since there are no reliable analytical theories available, the results of the PIC approach will be benchmarked against results from a numerical method (here called 'Legendre approach') using a decomposition of the perturbed distribution function into eigenfunctions of the pitch-angle scattering operator, which are Legendre polynomials.

\section{THEORY AND ALGORITHMS}

The theoretical description of kinetic Alfvén waves in a slab starts with the gyro-kinetic equation for the electrons

$$
\frac{\partial f_{\mathrm{e}}}{\partial t}+\dot{\mathbf{R}} \cdot \nabla f_{\mathrm{e}}+\dot{v}_{\|} \frac{\partial f_{\mathrm{e}}}{\partial v_{\|}}=\mathcal{L}\left(f_{\mathrm{e}}\right)
$$

in its $v_{\|}$-form. For simplicity, gyro-averaging and the dynamics of the ions have been neglected. The ions just provide a uniform background charge density to satisfy quasineutrality. $f_{\mathrm{e}}=f_{\mathrm{e}}(\mathbf{R}, \mathbf{v})$ is the electron distribution function, $\mathbf{R}$ denotes the spatial position, and $v_{\|}=\mathbf{b} \cdot \mathbf{v}$ is the velocity along the magnetic field $\mathbf{B}=B \mathbf{b}$. To treat the collisional influence on the distribution function, a pitch-angle $\left(\xi=v_{\|} / v\right)$ collision operator

$$
\mathcal{L}=\frac{\nu}{2} \frac{\partial}{\partial \xi}\left(1-\xi^{2}\right) \frac{\partial}{\partial \xi}
$$

is used. This operator follows from transforming the full Fokker-Planck operator to guiding-center coordinates (see e.g. Ref. [15]) followed by neglecting energy and guiding-center diffusion. For the electron-electron pitch-angle collisions considered here, this is well justified.

In the collision operator, $v$ is the electron self-collision frequency [14]

$$
v=v_{0} \frac{\Phi(x)-G(x)}{x^{3}}, \quad x=\frac{v}{\sqrt{2} v_{\text {th, }}},
$$

where $\Phi$ and $G$ denote the error function and the Chandrasekhar function, respectively. $\left(v_{\text {th,e }}=\sqrt{k_{\mathrm{B}} T_{\mathrm{e}} / m_{\mathrm{e}}}\right.$ is the electron thermal velocity.)

The characteristics of the kinetic equation are

$$
\begin{aligned}
\dot{\mathbf{R}} & =v_{\|} \mathbf{b}+\frac{1}{B_{\|}^{\star}} \mathbf{b} \times \nabla\left[\phi-v_{\|} A_{\|}\right] \\
\dot{v}_{\|} & =-\frac{q_{\mathrm{e}}}{m_{\mathrm{e}}}\left(\nabla_{\|} \phi+\frac{\partial A_{\|}}{\partial t}\right)
\end{aligned}
$$

with

$$
\begin{aligned}
\mathbf{B}^{\star} & =\nabla \times \mathbf{A}^{\star} \\
\mathbf{A}^{\star} & =\mathbf{A}_{0}+\left(\frac{m_{\mathrm{e}}}{q_{\mathrm{e}}} v_{\|}+A_{\|}\right) \mathbf{b}
\end{aligned}
$$


in a straight and constant magnetic field. (Here, $q_{\mathrm{e}}$ and $m_{\mathrm{e}}$ are the electron charge and mass, and $\mathbf{A}_{0}$ denotes the fixed background vector potential, respectively.) The electrostatic potential $\phi$ and the parallel component of the vector potential $A_{\|}$are denoted as usual.

Eqs. (1), (4), and (5) are solved together with the gyro-kinetic Poisson equation

$$
-\nabla \cdot\left(\frac{m_{\mathrm{i}} n_{0}}{B^{2}} \nabla_{\perp} \phi\right)=\left(1-\nabla \cdot \rho_{\mathrm{i}}^{2} \nabla_{\perp}\right) q_{\mathrm{e}} n_{\mathrm{e}}
$$

(with $\rho_{\mathrm{i}}=\sqrt{k_{\mathrm{B}} T_{\mathrm{i}} m_{\mathrm{i}}} /\left(q_{\mathrm{i}} B\right)$ being the ion gyro-radius) and Ampère's law

$$
-\nabla_{\perp}^{2} A_{\|}=\mu_{0} j_{\|, \mathrm{e}}
$$

for the fields. Since collisions are expected to mainly influence high $k_{\perp} \rho_{\mathrm{i}}$ modes, a Padé approximation for the ion polarization density was employed in Eq. (8). In the equations above, $m_{\mathrm{i}}$ is the ion mass, $n_{0}$ denotes the background density, $\mu_{0}$ is the vacuum permeability, and $j_{\|}$stands for the parallel current density. The electron density and current are obtained by taking the appropriate moments of the distribution function

$$
\begin{aligned}
n_{\mathrm{e}}(\mathbf{R}) & =\int \mathrm{d}^{3} v f_{\mathrm{e}}(\mathbf{R}, \mathbf{v}) \\
j_{\|, \mathrm{e}}(\mathbf{R}) & =q_{\mathrm{e}} \int \mathrm{d}^{3} v v_{\|} f_{\mathrm{e}}(\mathbf{R}, \mathbf{v}) .
\end{aligned}
$$

The kinetic equation together with the field equations will be solved in two different ways. Firstly, a particle-in-cell (PIC) method will be employed. Numerical marker particles, which move along the characteristics (Eqs. (4) and (5)) through phase-space, are used to represent the actual electrons. Secondly, the equations will be solved by employing a decomposition of the distribution function into Legendre polynomials ('Legendre approach'), which are eigenfunctions of the collision operator $\mathcal{L}$. A similar strategy was recently used in Ref. [1] for an electrostatic system.

The following subsections elaborate on the individual approaches.

\section{A. A short description of the pullback transformation scheme}

The PT-scheme was introduced in Refs. $[18,22]$ in the absence of collisions. Since those papers give a good overview of the general idea and of the implementation into a PIC code, only the key elements are repeated here. The implementation [16] of pitch-angle collisions into EUTERPE is discussed briefly.

The PT-scheme is based on an arbitrary splitting of $A_{\|}$into a Hamiltonian and a symplectic part, $A_{\|}=A_{\|}^{\mathrm{h}}+A_{\|}^{\mathrm{s}}$. Furthermore, an 'incomplete' transformation

$$
\tilde{u}_{\|}=v_{\|}+\frac{q_{\mathrm{e}}}{m_{\mathrm{e}}} A_{\|}^{\mathrm{h}}
$$

is employed. This transformation is similar to the one transforming to the canonical momentum $p_{\|}$, but 'incomplete' in the sense that it only contains the Hamiltonian part 
of $A_{\|}$. The resulting equations of motion are

$$
\begin{aligned}
\dot{\mathbf{R}}= & \left(\tilde{u}_{\|}-\frac{q_{\mathrm{e}}}{m_{\mathrm{e}}} A_{\|}^{\mathrm{h}}\right) \mathbf{b} \\
& +\frac{1}{B_{\|}^{\star}} \mathbf{b} \times \nabla\left[\phi-\tilde{u}_{\|}\left(A_{\|}^{\mathrm{h}}+A_{\|}^{\mathrm{s}}\right)\right] \\
\dot{\tilde{u}}_{\|}= & -\frac{q_{\mathrm{e}}}{m_{\mathrm{e}}}\left\{\nabla_{\|}\left[\phi-\tilde{u}_{\|} A_{\|}^{\mathrm{h}}\right]+\frac{\partial A_{\|}^{\mathrm{s}}}{\partial t}\right\}
\end{aligned}
$$

with

$$
\mathbf{A}^{\star}=\mathbf{A}_{0}+\left(\frac{m_{\mathrm{e}}}{q_{\mathrm{e}}} \tilde{u}_{\|}+A_{\|}^{\mathrm{s}}\right) \mathbf{b}
$$

for a plasma slab with constant magnetic field. While the Poisson equation for the PT-scheme is the same as Eq. (8), Ampère's law changes to

$$
-\nabla \cdot \nabla_{\perp} A_{\|}^{\mathrm{h}}+A_{\|}^{\mathrm{h}} \frac{\mu_{0} n_{0} q_{\mathrm{e}}^{2}}{m_{\mathrm{e}}}=\mu_{0} j_{\|, \mathrm{e}}+\nabla \cdot \nabla_{\perp} A_{\|}^{\mathrm{s}} \cdot
$$

It is important to note that, because of the arbitrary splitting of $A_{\|}$, an additional equation is needed to close the system. One thus gains an additional degree of freedom to fix $A_{\|}^{\mathrm{s}}$. This is done by postulating the equation

$$
\frac{\partial A_{\|}^{\mathrm{s}}}{\partial t}+\mathbf{b} \cdot \nabla \phi=0,
$$

which is similar to the ideal Ohm's law from MHD (magneto-hydrodynamic) theory. The idea is that, when simulating MHD modes (such as shear Alfvén waves) the majority of $A_{\|}$will be contained in $A_{\|}^{\mathrm{s}}$. Hence, $A_{\|}^{\mathrm{h}}$ will be small. In this way the cancellation problem is mitigated.

In the simulation, $A_{\|}^{\mathrm{h}}$ is chosen to be zero at $t=0$. However, as the simulation proceeds, $A_{\|}^{\mathrm{h}}$ increases, which could eventually lead to the reappearance of the cancellation problem. Therefore, after each time step, a resetting procedure (the actual pullback transformation) is performed: Before $A_{\|}^{\mathrm{h}}$ is reset to zero its value is added to $A_{\|}^{\mathrm{s}}$, Eq. (12) is used to go back from $\tilde{u}_{\|}$to $v_{\|}$, and the distribution function is transformed $f_{\mathrm{e}}\left(v_{\|}\right)=f_{\mathrm{e}}\left(\tilde{u}_{\|}\right)$. Thus, at its core, the PT-scheme is a method for solving the gyro-kinetic equations in the $v_{\|}$-formulation. As the collision operator is given in $v_{\|}$, the PT-scheme should be well suited to incorporate collisions.

In EUTERPE, the collision step is performed after the advection step, in which a fourthorder Runge-Kutta is used for time integration. The collisions themselves change the pitch-angle and magnetic moment of each particle. This involves rotating the velocity vector on a spherical shell (the magnitude of the velocity remains unchanged) according to randomly generated numbers with specific statistical properties. Denoting the cosine of the pitch-angle before and after the collision by $\xi_{\text {in }}$ and $\xi_{\text {out }}$ one finds

$$
\xi_{\text {out }}=\sin \chi \sin \lambda \sqrt{1-\xi_{\text {in }}^{2}}+\xi_{\text {in }} \cos \chi
$$


where $\chi=R \sqrt{2 v \Delta t}$ [16]. Both $\lambda$ and $R$ are random numbers. $\lambda$ is drawn from a uniform distribution between 0 and $2 \pi$, while $R$ comes from a Gaussian distribution with expectation value 0 and variance 1 [16]. This scheme was reported in Refs. [6, 28] and can be regarded as a variant of the method presented in Ref. [25]. It is important to note that this particular collisional implementation corresponds to a first-order (weak convergence) scheme. The often-used Euler-Maruyama method (see e.g. Ref. [19]) for the integration of stochastic differential equations is also only of first order.

\section{B. Solving the system by a decomposition into Legendre polynomials}

While EUTERPE solves the kinetic equation in the mixed formalism, the $p_{\|}$-formalism will be used for the Legendre approach. This means that, in contrast to Eqs. (4) and (5), the characteristics of the kinetic equation are now given as

$$
\begin{aligned}
\dot{\mathbf{R}} & =\left(u_{\|}-\frac{q_{\mathrm{e}}}{m_{\mathrm{e}}} A_{\|}\right) \mathbf{b}+\frac{1}{B_{\|}^{\star}} \mathbf{b} \times \nabla \psi \\
\dot{u}_{\|} & =-\frac{q_{\mathrm{e}}}{m_{\mathrm{e}}} \nabla_{\|} \psi \\
\psi & =\phi-u_{\|} A_{\|}
\end{aligned}
$$

with

$$
\mathbf{A}^{\star}=\mathbf{A}_{0}+\frac{m_{\mathrm{e}}}{q_{\mathrm{e}}} u_{\|} \mathbf{b}
$$

Note that in this formalism, $u_{\|}=p_{\|} / m_{\mathrm{e}}=v_{\|}+\left(q_{\mathrm{e}} / m_{\mathrm{e}}\right) A_{\|}$is not the physical velocity along the magnetic field lines.

Using the left-hand side of Eq. (1) in its $p_{\|}$-form and transforming it via

$$
u=\sqrt{u_{\|}^{2}+2 \mu B}, \quad \xi^{\prime}=\frac{u_{\|}}{u}
$$

to a $\left(u, \xi^{\prime}\right)$-coordinate system ( $\mu$ denotes the magnetic moment per mass) yields

$$
\frac{\partial f_{\mathrm{e}}^{(1)}}{\partial t}+u \xi^{\prime} \nabla_{\|} f_{\mathrm{e}}^{(1)}-\frac{q_{\mathrm{e}}}{m_{\mathrm{e}}} \xi^{\prime} \nabla_{\|} \psi \frac{\partial F}{\partial u}=\mathcal{L}(F)+\mathcal{L}\left(f_{\mathrm{e}}^{(1)}\right) .
$$

The ansatz $f_{\mathrm{e}}=F+f_{\mathrm{e}}^{(1)}$, with $F$ being the uniform Maxwellian background and $f_{\mathrm{e}}^{(1)}$ denoting a small perturbation, has also been used to linearize the kinetic equation. The $\nabla F$-term vanishes, since there are no gradients in density and temperature.

Note that $\mathcal{L}$ still acts in $(v, \xi)$-space. The term with the collision operator acting on the Maxwellian background is important as $F$ has to be transformed from $u_{\|}$to $v_{\|}$as will be discussed below. With a plane-wave ansatz $f_{\mathrm{e}}^{(1)} \sim \exp \left(\mathrm{i} k_{\|} z\right)$ the kinetic equation becomes

$$
\frac{\partial f_{\mathrm{e}}^{(1)}}{\partial t}+u \xi^{\prime} \mathrm{i} k_{\|} f_{\mathrm{e}}^{(1)}=-\frac{q_{\mathrm{e}}}{m_{\mathrm{e}}} \frac{u}{v_{\mathrm{th}, \mathrm{e}}^{2}} \xi^{\prime} F \mathrm{i} k_{\|} \psi+\mathcal{L}(F)+\mathcal{L}\left(f_{\mathrm{e}}^{(1)}\right)
$$


using the representation

$$
f_{\mathrm{e}}^{(1)}=\sum_{l=0}^{N_{l}} f_{l}(u, t) P_{l}\left(\xi^{\prime}\right) F,
$$

where $P_{l}$ is the $l$ th Legendre polynomial, and the $f_{l}$ are coefficients of the decomposition which have yet to be determined. One arrives at

$$
\begin{aligned}
\sum_{l} \frac{\partial f_{l}}{\partial t} P_{l} F & +u \xi^{\prime} \mathrm{i} k_{\|} \sum_{l} f_{l} P_{l} F= \\
& -\frac{q_{\mathrm{e}}}{m_{\mathrm{e}}} \frac{u}{v_{\mathrm{th}, \mathrm{e}}^{2}} \xi^{\prime} F \mathrm{i} k_{\|} \psi+\mathcal{L}(F)+\sum_{l} f_{l} F \mathcal{L}\left(P_{l}\right)
\end{aligned}
$$

where it still must be specified how the collision operator acts on the Maxwellian. In the $p_{\|}$-formulation $F$ is given as

$$
\begin{aligned}
F & =\frac{n_{0}}{\left(2 \pi v_{\mathrm{th}, \mathrm{e}}^{2}\right)^{3 / 2}} \exp \left(-\frac{u^{2}}{2 v_{\mathrm{th}, \mathrm{e}}^{2}}\right) \\
& \cong \frac{n_{0}}{\left(2 \pi v_{\mathrm{th}, \mathrm{e}}^{2}\right)^{3 / 2}} \exp \left(-\frac{v^{2}+2 v \xi\left(q_{\mathrm{e}} / m_{\mathrm{e}}\right) A_{\|}}{2 v_{\mathrm{th}, \mathrm{e}}^{2}}\right) .
\end{aligned}
$$

Hence,

$$
\begin{aligned}
\mathcal{L}(F) & =\frac{v}{2} \frac{\partial}{\partial \xi}\left(1-\xi^{2}\right) \frac{\partial F}{\partial \xi} \cong v \frac{q_{\mathrm{e}}}{m_{\mathrm{e}}} \frac{v \xi}{v_{\mathrm{th}, \mathrm{e}}^{2}} A_{\|} F \\
& \cong v \frac{q_{\mathrm{e}}}{m_{\mathrm{e}}} \frac{u \xi^{\prime}}{v_{\mathrm{th}, \mathrm{e}}^{2}} A_{\|} F
\end{aligned}
$$

where terms quadratic in $A_{\|}$have been neglected. Finally, this transformation of the Maxwellian leads to a correction proportional to the collision frequency and $A_{\|}$, a fact already reported in Ref. [29].

Next, the collision operator acting on the perturbation, $\mathcal{L}\left(f_{\mathrm{e}}^{(1)}\right)$, is transformed from $\xi$ to $\xi^{\prime}$ neglecting all non-linear terms in $A_{\|}$and $f_{\mathrm{e}}^{(1)}$. The result has the same form as the original $\mathcal{L}$ but with $\xi$ replaced by $\xi^{\prime}$.

In summary, one thus arrives at a linear equation fully acting in $\left(u, \xi^{\prime}\right)$-space.

Eq. (27) is now multiplied by $P_{l^{\prime}}$ and integrated over the pitch-angle variable $\left(\xi^{\prime} \in\right.$ $[-1,1])$. In this process the orthogonality relations of the Legendre polynomials are used. After some straightforward algebra one finds the final result

$$
\begin{aligned}
\frac{\partial f_{l}}{\partial t} & +\mathrm{i} k_{\|} u\left(\frac{l+1}{2 l+3} f_{l+1}+\frac{l}{2 l-1} f_{l-1}\right)=v \frac{q_{\mathrm{e}}}{m_{\mathrm{e}}} \frac{u}{v_{\mathrm{th}, \mathrm{e}}^{2}} A_{\|} \delta_{l, 1} \\
& -\frac{v}{2} l(l+1) f_{l}-\frac{q_{\mathrm{e}}}{m_{\mathrm{e}}} \mathrm{i} k_{\|} \frac{u}{v_{\mathrm{th}, \mathrm{e}}^{2}}\left[\phi \delta_{l, 1}-\frac{u A_{\|}}{3}\left(\delta_{l, 0}+2 \delta_{l, 2}\right)\right] .
\end{aligned}
$$


This is the electromagnetic extension of a very similar equation recently derived in Ref. [1].

The first term on the right-hand side of the above equation is the correction described by Eq. (29), the second and the third term result from the pitch-angle scattering operator and the parallel electric field, respectively.

An expression very similar to Eq. (30) can also be obtained for a simple Krook-type [3] collision operator $\mathcal{C}$. In that particular case the numerical implementation of Eq. (30) and the correctness of its solution could be verified, since the dispersion relation can be solved analytically. It is noteworthy to mention that only in the $v_{\|}$-formulation the Krook operator has the simple $\mathcal{C}_{v_{\|}}=-v f_{\mathrm{e}}^{(1)}$ form. In the $p_{\|}$-formulation, however, the Krook operator still has to relax the whole distribution function towards a Maxwellian defined with the physical parallel velocity. This gives rises to a (linearized) correction,

$$
\mathcal{C}_{p_{\|}}=-v f_{\mathrm{e}}^{(1)}+v \frac{q_{\mathrm{e}}}{m_{\mathrm{e}}} \frac{u_{\|}}{v_{\mathrm{th}, \mathrm{e}}^{2}} A_{\|} F
$$

in the $p_{\|}$-formulation, which is the same as the one associated with the pitch-angle collision operator.

The initial condition for Eq. (30) is chosen as $f_{0}(t=0)=1, f_{l}(t=0)=0$ for $l>1$, which corresponds to $f_{\mathrm{e}}^{(1)}(t=0)=F$.

The field equations for $\phi$ and $A_{\|}$in the $p_{\|}$-formulation, using a Padé approximation, are

$$
\frac{m_{i} n_{0}}{B^{2}} \frac{k_{\perp}^{2}}{1+k_{\perp}^{2} \rho_{\mathrm{i}}^{2}} \phi=q_{\mathrm{e}} n_{\mathrm{e}}, \quad\left(k_{\perp}^{2}+\frac{\mu_{0} n_{\mathrm{e}} q_{\mathrm{e}}^{2}}{m_{\mathrm{e}}}\right) A_{\|}=\mu_{0} j_{\|, \mathrm{e}} .
$$

The second term on the left-hand side of Ampère's law is the skin term only present in the $p_{\|}$-formulation. (Consequently, this term does not appear in Eq. (9).)

In Eq. (30) the variable $u$ is simply a parameter, which will be defined on an equidistant grid in the numerical implementation. To obtain $n_{\mathrm{e}}$ and $j_{\|, e}$ integrals over $\left(u, \xi^{\prime}\right)$-space have to be performed. The results are

$$
\begin{aligned}
n_{\mathrm{e}} & =4 \pi \int \mathrm{d} u u^{2} f_{0} F \\
j_{\|, \mathrm{e}} & =\frac{4 \pi}{3} q_{\mathrm{e}} \int \mathrm{d} u u^{3} f_{1} F .
\end{aligned}
$$

\section{CASE DEFINITION}

A plasma slab $(x, y, z)$ is simulated, with the magnetic field pointing in the $z$-direction and periodic boundary conditions applied in the $x$ - and $y$-directions. EUTERPE was built to work in geometries topologically equivalent to a torus, hence also the slab has to be treated as if it were a torus. In the following, the usual nomenclature for a toroidal system will be used, i.e. $(x, y, z)$ correspond to the radial, poloidal and toroidal coordinates $(r, \theta, \varphi)$.

All background quantities, such as temperatures, densities, and the magnetic field are chosen to be constant. The slab has a length $L=2 \pi \mathrm{m}$ in $z$. Its cross-section is quadratic 
Table I: Plasma parameters

\begin{tabular}{l|l}
\hline \hline magnetic field $B / \mathrm{T}$ & 1.0 \\
\hline electron density $n_{\mathrm{e}} / \mathrm{m}^{-3}$ & $1.037 \cdot 10^{19}$ \\
\hline ion density $n_{\mathrm{i}} / \mathrm{m}^{-3}$ & $n_{\mathrm{e}}$ \\
\hline electron temperature $T_{\mathrm{e}} / \mathrm{keV}$ & 9.579 \\
\hline ion temperature $T_{\mathrm{i}} / \mathrm{keV}$ & $20 T_{\mathrm{e}}$ \\
\hline gyration frequency $\Omega_{\mathrm{i}} / \mathrm{s}^{-1}$ & $9.579 \cdot 10^{7}$ \\
\hline \hline
\end{tabular}

with the length of one side being $\Delta=1.4 \mathrm{~m}$. The parallel and the perpendicular wavevector, expressed through the toroidal and poloidal Fourier numbers $n$ and $m$ and the radial mode number $o$, are given by

$$
\begin{aligned}
& k_{\|}=\frac{2 \pi n}{L}=\frac{n}{100 \rho_{\mathrm{s}}} \\
& k_{\perp}^{2}=k_{r}^{2}+k_{\theta}^{2}=\frac{4 \pi^{2}}{\Delta^{2}}\left(o^{2}+m^{2}\right)=\frac{4 \pi^{2}}{140^{2} \rho_{\mathrm{s}}^{2}}\left(o^{2}+m^{2}\right)
\end{aligned}
$$

with the normalization quantity $\rho_{\mathrm{s}}=1 \mathrm{~cm}\left(\rho_{\mathrm{s}}=\sqrt{k_{\mathrm{B}} T_{\mathrm{e}} m_{\mathrm{i}}} /\left(q_{\mathrm{i}} B\right)\right.$ denotes the sound gyro-radius). Slightly different kinetic Alfvén waves with a fixed value $n=1$, but varying in their poloidal mode number $m$ are considered in the numerical simulations. The radial mode number is chosen as $o=1 / 2$, because the initial perturbation used in the EUTERPE runs is a half sine-wave in $r$. Several other parameters of this case are listed in Tab. I. If collisions are used, the velocity-independent part of the collision frequency is $v_{0}=0.15 \Omega_{\mathrm{i}}$ (with the ion gyration frequency $\Omega_{\mathrm{i}}=q_{\mathrm{i}} B / m_{\mathrm{i}}$ ).

All EUTERPE calculations are performed using a phase-factor, $\exp (\mathrm{i} m \theta+\mathrm{i} n \varphi)$, to extract the dominant poloidal and toroidal Fourier harmonics from all perturbed quantities. The spatial resolution was chosen as $n_{r}=16, n_{\theta}=8$, and $n_{\varphi}=1$ (parallel direction). This leads to a radial resolution of $8.75 \mathrm{~cm}$. This is circa 50 times larger than the electron skin depth $\delta_{\mathrm{e}}=\rho_{\mathrm{s}} \sqrt{2 m_{\mathrm{e}} /\left(m_{\mathrm{i}} \beta_{\mathrm{e}}\right)}$, which does not need to be resolved in the EUTERPE simulations. ( $\beta_{\mathrm{e}}$ is the electron plasma beta defined as usual.)

For the Legendre approach, $f_{\mathrm{e}}^{(1)}$ is decomposed into the first 20 (40 to obtain Fig. 4) Legendre polynomials. As for the EUTERPE simulations, a fourth-order Runge-Kutta method is used for the time-integration. The time step is $\Delta t \Omega_{\mathrm{i}}=0.02$ (0.005 to obtain Fig. 4). The integration over $u$-space is performed on an equidistant grid (400 grid points) with $u_{\max } \approx 9.33 v_{\text {th,e }}$ using the trapezoidal rule. All results are converged with respect to those parameters. 

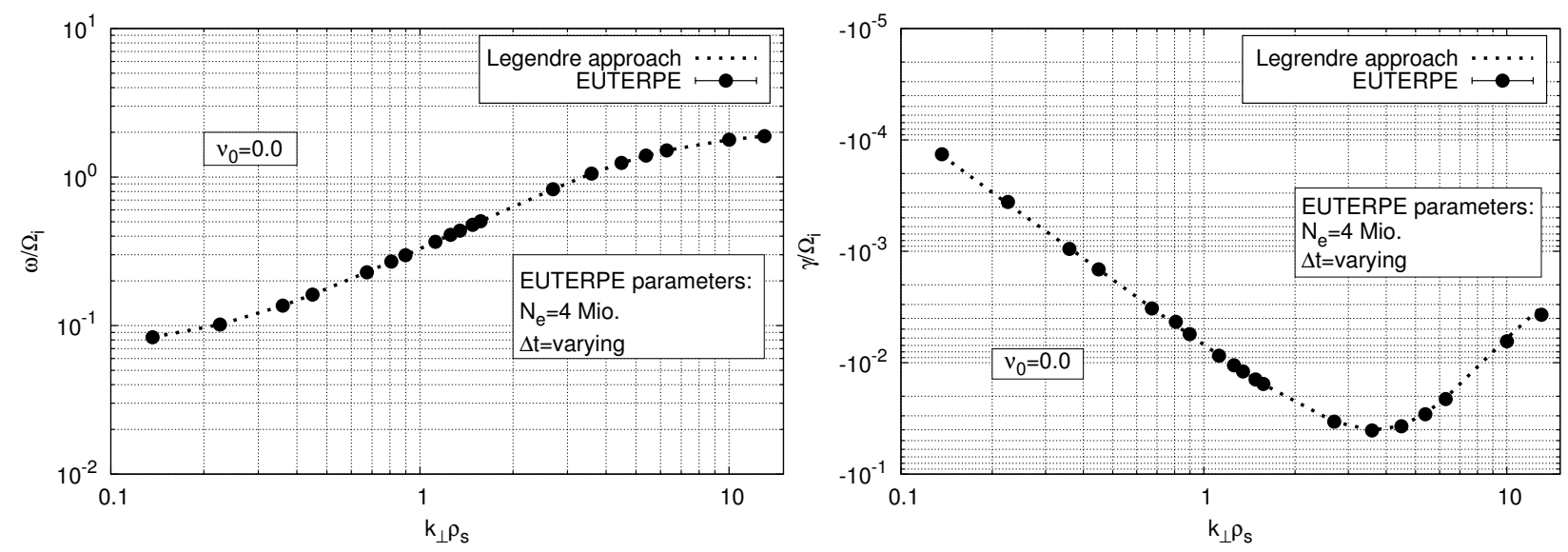

Figure 1: Real frequency (left) and damping rate (right) of the kinetic Alfvén wave without collisions. EUTERPE and the decomposition of the perturbed electron distribution function into Legendre polynomials ('Legendre approach') yield virtually the same result.

\section{RESULTS}

\section{A. Collisional influences on the dispersion relation}

In the case of the EUTERPE simulations, the kinetic equation, Eq. (1), is solved by a PICmethod. The equations of motion (see Eqs. (13) and (14)) are advanced in time using a fourth-order Runge-Kutta method. At each time step, the field equations (8) and (16) are solved.

A very similar strategy is pursued for the Legendre approach, where the modified kinetic equation (30) is instead solved together with the field equations given by Eq. (32). The real frequency $\omega$ and the damping rate $\gamma$ of the mode can be seen in Fig. 1 for $v_{0}=0$. Different mode numbers $m$, corresponding to different $k_{\perp} \rho_{\mathrm{s}}$, have been used to obtain this dispersion relation.

For $k_{\perp} \rho_{\mathrm{s}} \gtrsim 3.5$ Fig. 1 shows frequencies that are larger than the ion cyclotron frequency which contradicts one of the basic assumptions of gyro-kinetics. However, since the focus of this paper is to verify the correctness of the numerical algorithm (and not to validate the physics) the benchmark can be performed in that parameter range to illustrate clearly the effect of collisions (see below).

As can be seen in the figure, the mode is Landau-damped with a maximum damping rate around $k_{\perp} \rho_{\mathrm{S}} \approx 3$. While $\gamma$ varies over three orders of magnitude, the change in $\omega$ is smaller. The dotted line corresponds to the Legendre approach. The full circles have been obtained by EUTERPE. As expected, the agreement between the two different approaches is very good.

Both numerical implementations have also been compared with analytical theory in the collision-less case. For $v_{0}=0$ it is straightforward to obtain the analytical dispersion relation

$$
1-\frac{m_{\mathrm{i}} \beta_{\mathrm{e}}}{m_{\mathrm{e}}} \frac{1}{k_{\perp}^{2} \rho_{\mathrm{s}}^{2}}\left\{\zeta^{2}-\frac{m_{\mathrm{e}}}{m_{\mathrm{i}} \beta_{\mathrm{e}}}\left(1+\frac{T_{\mathrm{i}}}{T_{\mathrm{e}}} k_{\perp}^{2} \rho_{\mathrm{s}}^{2}\right)\right\}[1+\zeta \mathrm{Z}(\zeta)]=0
$$



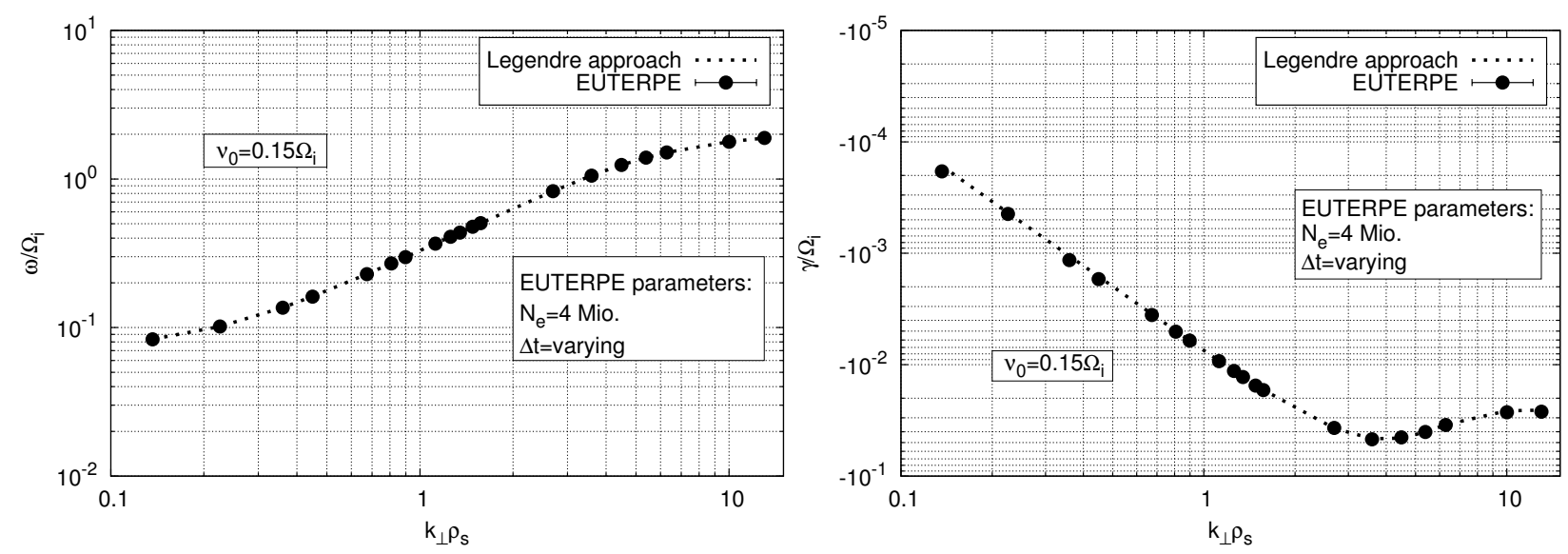

Figure 2: Real frequency (left) and damping rate (right) of the kinetic Alfvén wave under the influence of collisions. The agreement between EUTERPE and the Legendre approach is still very good. Collisional damping mainly affects large $k_{\perp} \rho_{\mathrm{s}}$. Compared with the case without collisions, the time step had to be reduced for small values of $k_{\perp} \rho_{\mathrm{s}}$.

that can be solved for $\zeta$ numerically. Here, $\zeta=(\omega+\mathrm{i} \gamma) /\left(\sqrt{2} k_{\|} v_{\text {th,e }}\right)$ and $Z$ denotes the plasma dispersion function. Both numerical approaches agree very well with Eq. (37) in the collision-less limit.

Having verified EUTERPE and the Legendre approach for $v_{0}=0$, a case where collisions are included $\left(v_{0}=0.15 \Omega_{\mathrm{i}}\right)$ may be investigated next. Note that, in particular, the implementation of collisions is very different in the Legendre approach and the PICmethod.

Fig. 2 shows how the dispersion relation changes if collisions are taken into account. While there is no noticeable change in the frequency $\omega$, the damping rate is modified substantially. $\gamma$ is now a combination of pure Landau damping and collisional damping. In comparison with Fig. 1, the mode is a little bit stronger damped due to collisional damping for small $k_{\perp} \rho_{\mathrm{s}}$. The largest differences, however, are seen at high $k_{\perp} \rho_{\mathrm{s}}>$ 4 , where the mode is now much stronger damped than before. Pitch-angle collisions randomize the velocity distribution to a certain degree and thus have a smoothing effect on the perturbations. It is thus not surprising that the collisions have a larger effect on the small scales, i.e. high $k_{\perp} \rho_{\mathrm{s}}$. Again, the agreement between the Legendre approach (dotted line) and EUTERPE (full circles) is very good for all values of $k_{\perp} \rho_{\mathrm{s}}$.

It usually takes several collision times until the effects of collisions become apparent in a numerical simulation. Thus, a relatively high $v_{0}$ was chosen. This leads to a short collision time $\tau_{\text {coll }}=1 / \nu$ and therefore to a reduced over-all time span required to be simulated.

A numerical study showing the effect of increasing collision frequency $v_{0}$ on the damping rate is presented in Fig. 3. This has been done for a fixed mode number $m=290$, which corresponds to $k_{\perp} \rho_{\mathrm{s}}=13$. Whereas the frequency stays roughly the same for different collision frequencies, the damping rate increases linearly with rising $v_{0}$. This is the expected behaviour as the collision operator $\mathcal{L}$ also scales linearly with $v_{0}$.

Apart from the influence on the damping rate of the kinetic Alfvén wave, the effect of collisions becomes most apparent when investigating the perturbed distribution func- 


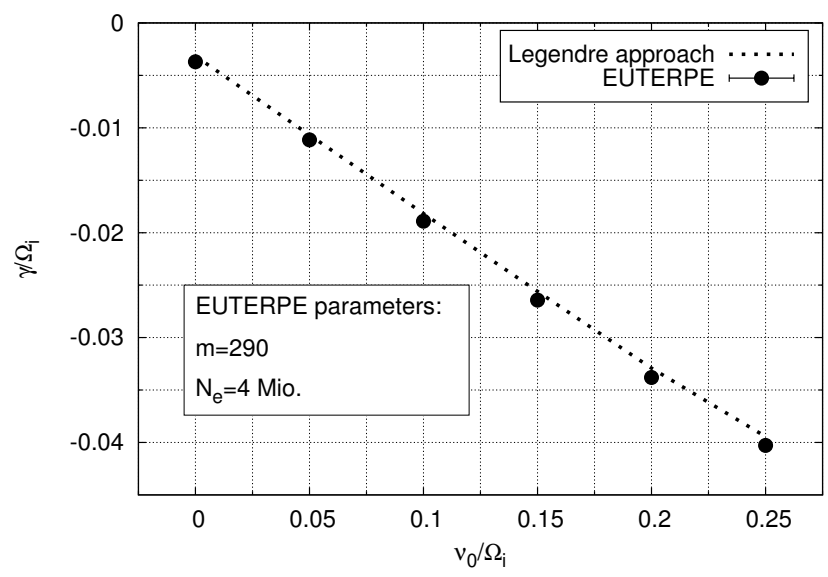

Figure 3: Influence of an increasing collision frequency on the damping rate of the kinetic Alfvén wave. While the frequency (not shown) stays almost the same, the damping rate scales linearly with $v_{0}$.

tion $f_{\mathrm{e}}^{(1)}$ in velocity-space $\left(u, \xi^{\prime}\right)$. Fig. 4 shows the results obtained by the Legendre approach. For this particular case the mode number $m=25$ has been used, corresponding to an intermediate $k_{\perp} \rho_{\mathrm{s}} \approx 1$.12. In Fig. $4, f_{\mathrm{e}}^{(1)}$ is plotted at different times in the simulation without (left-hand side) and with (right-hand side) collisions. In all diagrams the resonance condition

$$
\omega-u_{\|} k_{\|}=0
$$

is indicated as a solid black line using the $\omega$ obtained from the simulation.

Starting from the same initial condition, which is a Maxwellian and therefore isotropic in $\xi^{\prime}$, it can be seen that collisions have a strong effect on the structure of $f_{\mathrm{e}}^{(1)}$. Without collisions the perturbed distribution function becomes highly non-isotropic in pitch-angle. $f_{\mathrm{e}}^{(1)}$ peaks around those velocities fulfilling Eq. (38), as expected. As time progresses, the structures in velocity-space become even finer.

When collisions are included, the velocity-space becomes more isotropic with respect to $\xi^{\prime}$ for $t \Omega_{\mathrm{i}} \geq 35$. The strong peaking of $f_{\mathrm{e}}^{(1)}$ around the resonant velocities has almost vanished, but the perturbed distribution function is still localized in the vicinity of the resonances. The small scales in velocity-space are effectively damped. Furthermore, the shape of $f_{\mathrm{e}}^{(1)}$ in velocity space seems to have quickly reached a nearly converged state, nevertheless the amplitude is growing slightly (less than in the collision-less case) between $t \Omega_{\mathrm{i}}=35$ and $t \Omega_{\mathrm{i}}=70$.

Another feature of collisions is related to the long-time behaviour of $f_{\mathrm{e}}^{(1)}$. Without collisions, the perturbed part of the distribution function never vanishes. The damping of the potentials $\phi$ and $A_{\|}$comes from the integration over velocity-space in combination with the developing fine-scale structure. Pitch-angle collisions, on the other hand, have the property of relaxing the distribution function towards a Maxwellian in this linear problem. Hence (because of the splitting $f_{\mathrm{e}}=F+f_{\mathrm{e}}^{(1)}$ applied to the distribution function), $f_{\mathrm{e}}^{(1)}$ has to vanish for $t \rightarrow \infty$. 

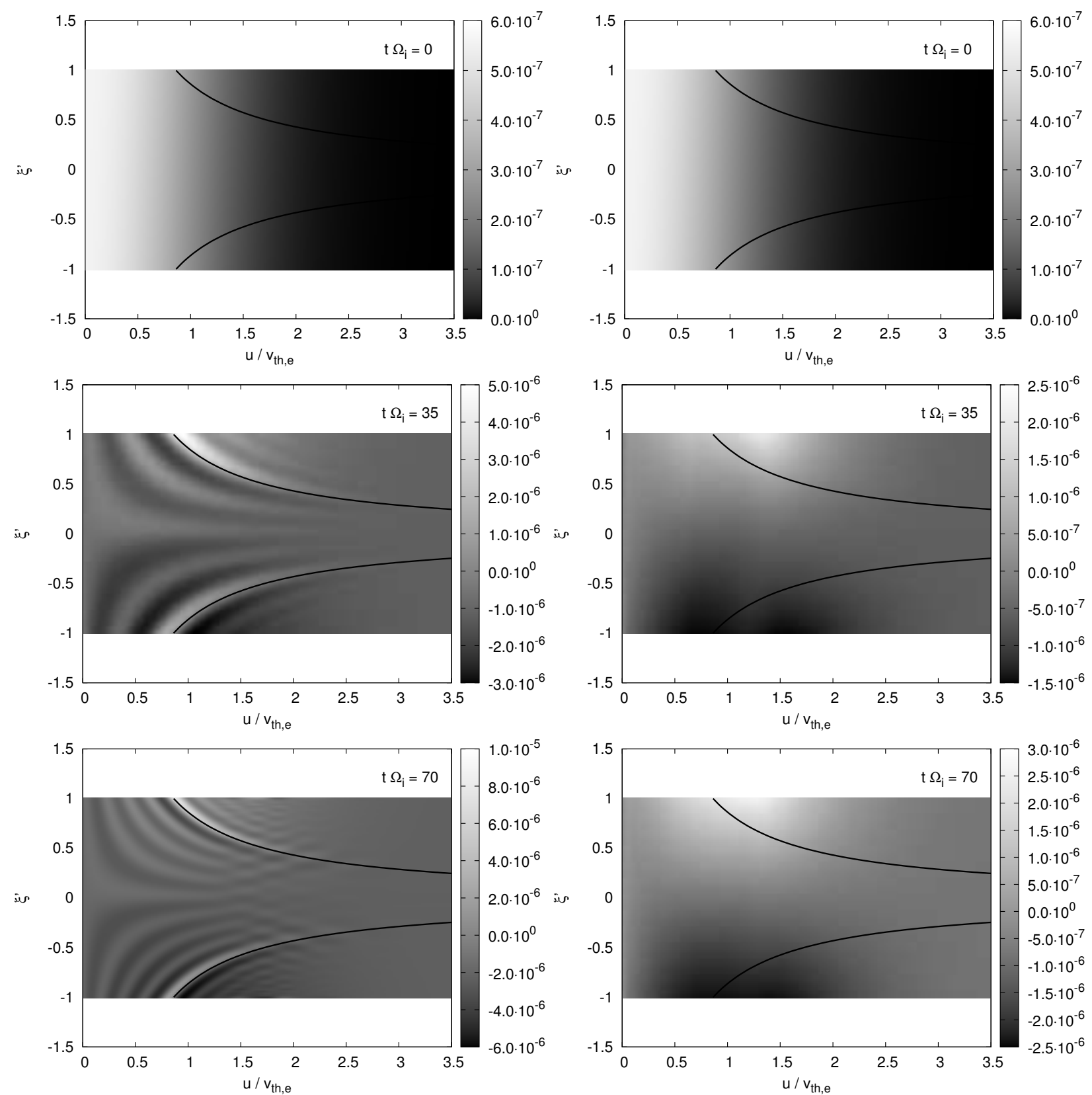

Figure 4: The perturbed distribution function (at $k_{\|} z=\pi / 4$ ) in velocity-space at different simulation times. Collisions are included only on the right-hand side. While $f_{\mathrm{e}}^{(1)}$ peaks around the resonant velocities (black line) without collisions, velocity-space becomes more isotropic in $\xi^{\prime}$ for the case including collisions. All solutions for $f_{\mathrm{e}}^{(1)}$ were obtained by the Legendre approach.

It was observed in long-time simulations (about five times longer than the longest time shown in Fig. 4) that $f_{\mathrm{e}}^{(1)}$ integrated over all velocities (using the $L^{2}$-norm) saturates at a finite value in the collision-less case, but vanishes if collisions are included. 

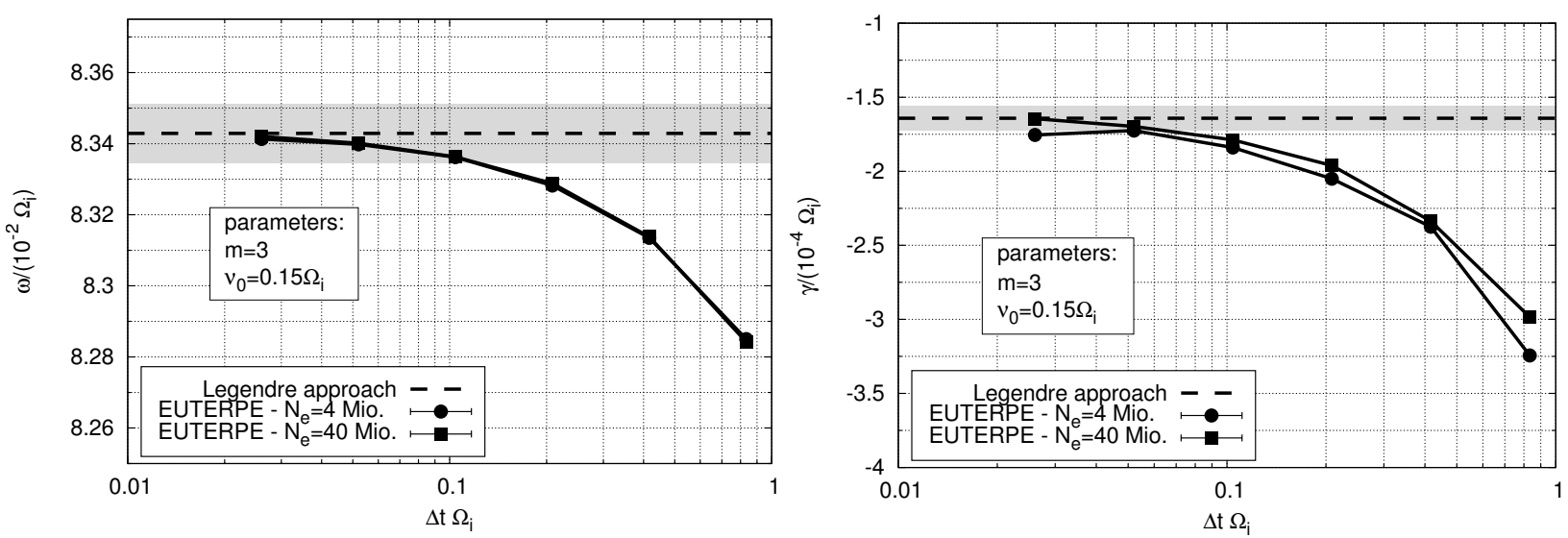

Figure 5: Convergence of the real frequency (left) and of the damping rate (right) determined by EUTERPE with decreasing time step under the influence of collisions. The gray region on the left-hand side corresponds to an uncertainty level of $0.1 \%$. Since it is more difficult to resolve the damping rate, the gray region indicates $5 \%$ uncertainty on the right-hand side. Since increasing the particle number by a factor of 10 leads to nearly the same behaviour, the convergence seems to be bound by $\Delta t$. The convergence with respect to $\Delta t$ is of first order.

\section{B. Convergence studies}

1. Convergence study for $\Delta t$ and small $k_{\perp} \rho_{\mathrm{s}}$

As can be seen in Figs. 1 and 2, the frequency of the mode increases with rising values of $k_{\perp} \rho_{\mathrm{s}}$. In order to fully resolve the ever-faster oscillatory behaviour, the time step had to be decreased with increasing values of the poloidal mode number $m$. Fig. 1 confirms that the time steps were chosen well for the cases without collisions. With collisions, however, $\Delta t$ had to be chosen smaller at low values of $k_{\perp} \rho_{\mathrm{S}}$ (at high values of $k_{\perp} \rho_{\mathrm{S}}$ the time step was already small enough). This is due to the fact that, when collisions are included in the EUTERPE simulations, a stochastic scheme is chosen. As this scheme only has a weak convergence of order one (in $\Delta t$ ) and is thus less accurate than the fourth-order Runge-Kutta, a smaller time step needs to be chosen.

A convergence scan with respect to the time step $\Delta t$ was performed for the EUTERPE calculation including collisions at $m=3\left(k_{\perp} \rho_{\mathrm{S}} \approx 0.14\right)$. Fig. 5 shows the results. Using the same time step $\Delta t \Omega_{\mathrm{i}} \approx 0.83$ with and without collisions does not lead to accurate results for $\omega$ and $\gamma$ in the collisional case: The damping rate is for instance wrong by a factor of two. This is due to the aforementioned change in the time-integrator from a fourth-order Runge-Kutta to a first-order stochastic method. Note that for this small value of $k_{\perp} \rho_{\mathrm{S}}$ collisions have nearly no influence on $\omega$ and $\gamma$ (see Fig. 2), but they still determine the time step.

However, Fig. 5 also shows how EUTERPE converges to the result obtained by the Legendre approach with decreasing values of $\Delta t$. Thus, when including collisions into the simulations, it should be carefully checked if the result is converged with respect to the time step. Especially since the numerical error of the integration scheme increases that drastically. 

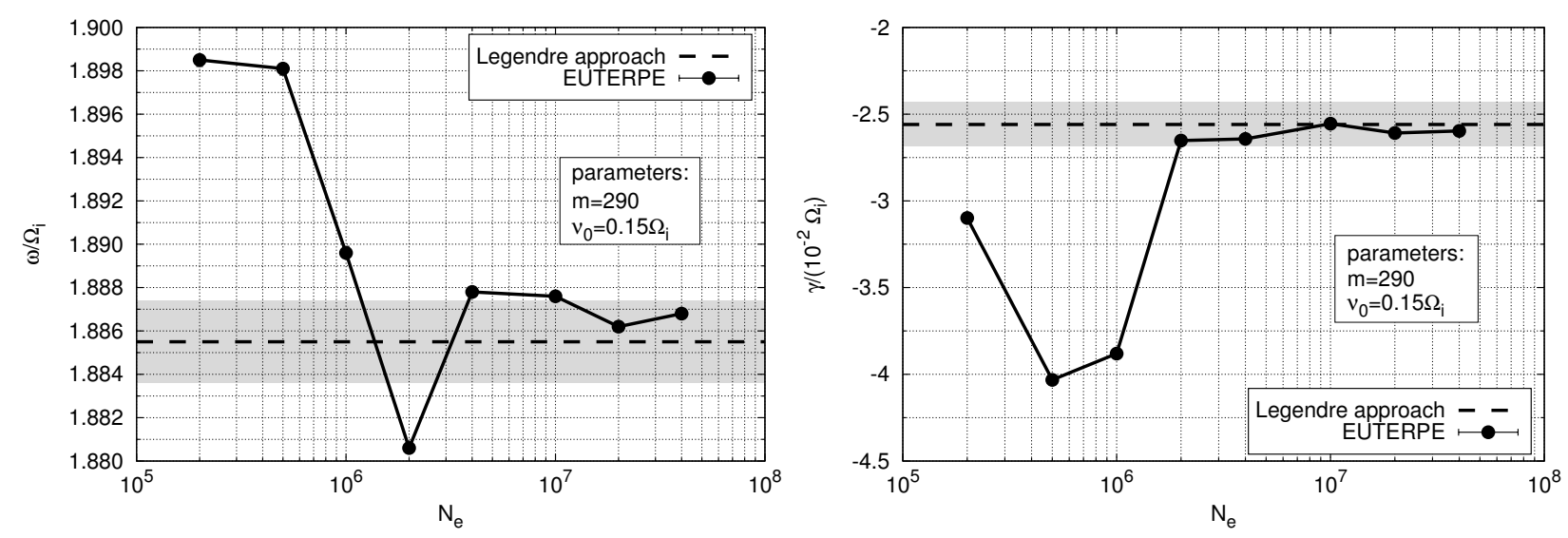

Figure 6: Convergence of the real frequency (left) and of the damping rate (right) with increasing number of electron markers in EUTERPE. Collisions are present. The uncertainty levels are the same as in Fig. 5.

\section{Convergence study for $N_{\mathrm{e}}$ and high $k_{\perp} \rho_{\mathrm{s}}$}

Figs. 2 and 3 nicely demonstrate the correct implementation of collisions in the electromagnetic version of EUTERPE. However, to obtain the results presented in Fig. 2 rather high particle numbers have been chosen in order to ensure numerically converged results. Now, a convergence study (for the last point of Fig. 2 at $k_{\perp} \rho_{\mathrm{S}}=13, m=290$ ) has been conducted with the aim to reduce the required particle number. Fig. 6 summarizes the results.

It can be seen that particle numbers greater than 4 million are not necessary for an accurate determination of $\omega$ and $\gamma$. Reducing the particle number by a factor of 2 to 2 million still gives a frequency that is approximately only $0.2 \%(\approx 5 \%$ for $\gamma)$ off compared with the Legendre approach. For particle numbers greater than 2 million the agreement between both approaches is very good. This is even less than the 4 million numerical marker particles used in the case without collisions (Fig. 1). This hints on the fact that not the optimum particle number may have been used in that case.

Seeing how on the left-hand side of Fig. 6 nearly all points scatter around the $0.1 \%$ uncertainty interval emphasizes again that the frequency is more robust than the damping rate for this case.

\section{Comparison of $p_{\|}$- and $v_{\|}$-formulation}

All the EUTERPE results have been obtained using the PT-scheme. For the second strategy presented in this paper, using the Legendre approach to solve the kinetic equation, the $p_{\|}$-formalism has been used exclusively so far. This avoids the numerically difficult $\partial A_{\|} / \partial t$-term inherent to the $v_{\|}$-formulation, but requires the introduction of a correction resulting from $\mathcal{L}(F)$. It is not required to change the collision operator (given in $\left.v_{\|}\right)$directly, because all corrections are non-linear in the perturbations and therefore negligible.

When comparing the results obtained with the Legendre approach with the EUTERPE 

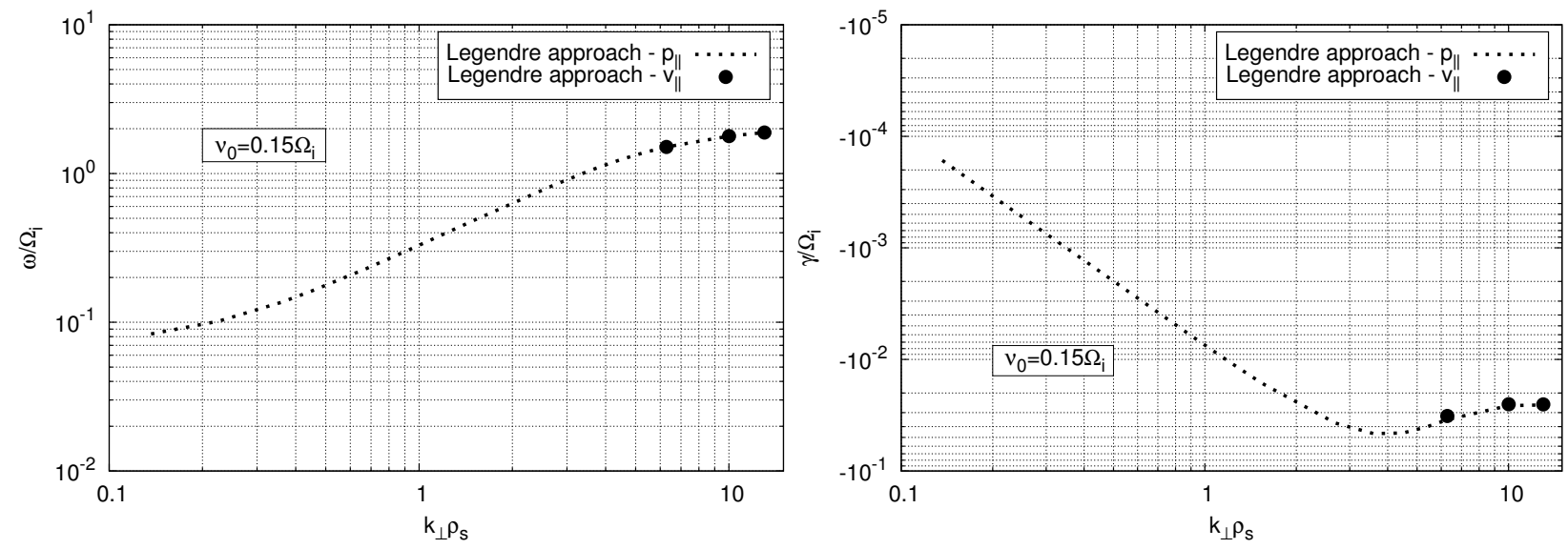

Figure 7: Comparison of the $p_{\|}$- and $v_{\|}$-formulation of the equations. Good agreement is found for values of $k_{\perp} \rho_{\mathrm{S}}$ for which the $v_{\|}$-formulation was numerically stable.

results it should be pointed out that the collision step in EUTERPE is performed while being in the $v_{\|}$-frame. It is therefore worthwhile to examine what happens to the Legendre approach when going from $p_{\|}$to $v_{\|}$.

This transition requires the $\dot{u}_{\|}$equation to be replaced by

$$
\dot{v}_{\|}=-\frac{q_{\mathrm{e}}}{m_{\mathrm{e}}}\left(\nabla_{\|} \phi+\frac{\partial A_{\|}}{\partial t}\right) .
$$

Consequently, the skin term in Ampère's law and $\mathcal{L}(F)$ do no longer appear. Hence, in the kinetic equation only the $A_{\|}$-terms change and Eq. (30) must be transformed to

$$
\begin{aligned}
& \frac{\partial}{\partial t}\left(f_{l}+\frac{q_{\mathrm{e}}}{m_{\mathrm{e}}} \frac{v}{v_{\mathrm{th}, \mathrm{e}}^{2}} A_{\|} \delta_{l, 1}\right)+\mathrm{i} k_{\|} v\left(\frac{l+1}{2 l+3} f_{l+1}+\frac{l}{2 l-1} f_{l-1}\right) \\
& =-\frac{v}{2} l(l+1) f_{l}-\frac{q_{\mathrm{e}}}{m_{\mathrm{e}}} \mathrm{i} k_{\|} \frac{v}{v_{\mathrm{th}, \mathrm{e}}^{2}} \phi \delta_{l, 1} .
\end{aligned}
$$

Contrary to the $p_{\|}$-formulation, $f_{1}$ is now integrated together with the vector potential, i.e.

$$
\tilde{f}_{1}=f_{1}+\frac{q_{\mathrm{e}}}{m_{\mathrm{e}}} \frac{v}{v_{\text {th,e }}^{2}} A_{\|}
$$

and after each Runge-Kutta sub-step $f_{1}$ is computed from $\tilde{f}_{1}$ using the $A_{\|}$from the previous step. This emphasizes that the $v_{\|}$-formulation is numerically indeed more challenging.

In Fig. 7 it is shown that also for electromagnetic simulations including collisions the agreement between the $p_{\|^{-}}$(dotted line) and $v_{\|}$-implementation (full circles) is very good. However, this comparison could only be carried out for three data points with high mode numbers, because the integration scheme became numerically unstable in $v_{\|}$for smaller values of $k_{\perp} \rho_{\mathrm{s}}$. Using an implicit scheme for the time integration might prevent the numerical instability. 


\section{SUMMARY AND CONCLUSIONS}

The applicability of the pullback transformation scheme [18, 22] to an electromagnetic system influenced by electron pitch-angle collisions has been investigated using the gyro-kinetic PIC code EUTERPE. For comparison, a decomposition of the perturbed electron distribution function into Legendre polynomials was used as a second strategy to solve the equations.

The widely used $p_{\|}$-approach has the drawback that the collisional and the noncollisional parts of the kinetic equation operate in different coordinates. This is counterintuitive and necessitates the introduction of a correction term. On the other hand, the PT-scheme, apart from its other advantages, operates in $v_{\|}$-space. Here we showed by comparing the PIC and the Legendre approach that this scheme is a natural choice for including collisional effects in electromagnetic PIC simulations and is compatible with a two-weight scheme.

After the successful benchmark between EUTERPE and the Legendre approach the effects of collisions on $f_{\mathrm{e}}^{(1)}$ were studied in greater detail. It was found that because of collisions, the velocity-space becomes much more isotropic with respect to the pitch-

angle. $f_{\mathrm{e}}^{(1)}$ is completely different with and without collisions, which might be the reason why perturbative theories on this topic are scarce.

All simulations have been performed in slab geometry in order to concentrate on the essential properties of the numerical scheme. The parameters of this case were chosen in a way to facilitate a straightforward comparison between EUTERPE and the Legendre approach with the focus of the benchmark being on verification rather than on validation.

The convergence scans showed that, when collisions are included, choosing a small time step is more important than having a high particle number. This could easily be understood as the integration scheme changes from a fourth-order Runge-Kutta to a first-order (weak convergence) stochastic scheme. For more complex systems with higher constraints on the time step, it could be worthwhile to test stochastic integrators of higher order.

For simplicity, only results excluding the ion dynamics have been presented in this paper. A fully kinetic extension is straightforward.

The consequent next steps would be performing simulations in more interesting parameter regimes and in more realistic geometries, e.g. in tokamaks or stellarators. Moreover, since only electron-electron pitch-angle collisions were considered so far, the collision operator should be extended accordingly.

\section{Acknowledgment}

The authors gratefully acknowledge helpful discussions with Per Helander and Alessandro Zocco.

A part of this work was carried out using the HELIOS supercomputer system at the Computational Simulation Centre of International Fusion Energy Research Centre (IFERC-CSC), Aomori, Japan, under the Broader Approach collaboration between 
Euratom and Japan, implemented by Fusion for Energy and JAEA.

This work has been carried out within the framework of the EUROfusion Consortium and has received funding from the Euratom research and training programme 20142018 under grant agreement No 633053. The views and opinions expressed herein do not necessarily reflect those of the European Commission. 


\section{References}

[1] J. W. Banks, S. Brunner, R. L. Berger, and T. M. Tran. Vlasov Simulations of Electron-Ion Collision Effects on Damping of Electron Plasma Waves . 2016. http://www.arxiv.org/ abs/1601.01002.

[2] H. L. Berk, B. N. Breizman, and H. Ye. Scenarios for the Nonlinear Evolution of AlphaParticle-Induced Alfvén Wave Instability. Physical Review Letters, 68:3563, 1992.

[3] P. L. Bhatnagar, E. P. Gross, and M. Krook. A Model for Collision Processes in Gases. I. Small Amplitude Processes in Charged and Neutral One-Component Systems. Physical Review Letters, 94:511-525, 1954.

[4] A. Bierwage, Y. Todo, N. Aiba, and K. Shinohara. Dynamics of low- $n$ shear Alfvén modes driven by energetic N-NB ions in JT-60U. Nuclear Fusion, 54:104001, 2014.

[5] A. J. Brizard and T. S. Hahm. Foundations of nonlinear gyrokinetic theory. Reviews of modern Physics, 79:421, 2007.

[6] S. Brunner, E. Valeo, and J. .A. Krommes. Collisional delta-f scheme with evolving background for transport time scale simulations. Physics of Plasmas, 6:4504, 1999.

[7] Y. Chen and S. E. Parker. A $\delta f$ particle method for gyrokinetic simulations with kinetic electrons and electromagnetic perturbations. Journal of Computational Physics, 189:463, 2003.

[8] G. Y. Fu, C. Z. Cheng, and K. L. Wong. Stability of the toroidicity-induced Alfvén eigenmode in axisymmetric toroidal equilibria. Physics of Fluids B: Plasma Physics, 5:4040, 1993.

[9] J. M. García-Regaña, C. D. Beidler, R. Kleiber, P. Helander, A. Mollén, J. A. Alonso, M. Landreman, H. Maaßberg, H. M. Smith, Y. Turkin, and L. Velasco. Electrostatic potential variation on the flux surface and its impact on impurity transport. Nuclear Fusion, 57:056004, 2017.

[10] J. M. García-Regaña, R. Kleiber, C. D. Beidler, H. Maaßberg, P. Helander, and Y. Turkin. On Neoclassical impurity transport in stellarator geometry. Plasma Physics and Controlled Fusion, 55:074008, 2013.

[11] N. N. Gorelenkov and S. E. Sharapov. On the Collisional Damping of TAE-modes on Trapped Electrons in Tokamaks. Physica Scripta, 45:163, 1992.

[12] T. S. Hahm, W. W. Lee, and A. Brizard. Nonlinear gyrokinetic theory for finite-beta plasmas. Physics of Fluids, 31:1940, 1988.

[13] R. Hatzky, A. Könies, and A. Mishchenko. Electromagnetic gyrokinetic PIC simulation with an adjustable control variates method. Journal of Computational Physics, 225:568, 2007.

[14] P. Helander and D. J. Sigmar. Collisional Transport in Magnetized Plasmas. Cambridge University Press, 2002.

[15] E. Hirvijoki, A. Brizard, A. Snicker, and T. Kurki-Suonio. Monte Carlo implementation of a guiding-center Fokker-Planck kinetic equation. Physics of Plasmas, 20:092505, 2013.

[16] K. Kauffmann. Including Collisions in Gyrokinetic Tokamak and Stellarator Simulations. PhD thesis, Ernst-Moritz-Arndt-Universität Greifswald, 2011.

[17] K. Kauffmann, R. Kleiber, R. Hatzky, and M. Borchardt. Global linear gyrokinetic simulations for LHD including collisions. Journal of Physics: Conference Series, 260:012014, 2010. 
[18] R. Kleiber, R. Hatzky, A. Könies, A. Mishchenko, and E. Sonnendrücker. An explicit large time step particle-in-cell scheme for nonlinear gyrokinetic simulations in the electromagnetic regime. Physics of Plasmas, 23:032501, 2016.

[19] P. E. Kloeden and E. Platen. Numerical Solution of Stochastic Differential Equations. Springer, 1999.

[20] J. Lang, G. Y. Fu, and Y. Chen. Nonlinear simulation of toroidal Alfvén eigenmode with source and sink. Physics of Plasmas, 17:042309, 2010.

[21] A. Mishchenko, M. Borchardt, M. Cole, R. Hatzky, T. Fehér, R. Kleiber, A. Könies, and A. Zocco. Global linear gyrokinetic particle-in-cell simulations including electromagnetic effects in shaped plasmas. Nuclear Fusion, 55:053006, 2015.

[22] A. Mishchenko, A. Könies, R. Kleiber, and M. Cole. Pullback transformation in gyrokinetic electromagnetic simulations. Physics of Plasmas, 21:092110, 2014.

[23] J. V. W. Reynders. Gyrokinetic simulations of finite- $\beta$ plasmas on parallel architectures. PhD thesis, Princeton University, 1992.

[24] E. Sonnendrücker, A. Wacher, R. Hatzky, and R. Kleiber. A split control variate scheme for PIC simulations with collisions. Journal of Computational Physics, 295:402, 2015.

[25] T. Takizuka and H. Abe. A binary collision model for plasma simulation with a particle code. Journal of Computational Physics, 25:205, 1977.

[26] D. J. Thuecks, C. A. Kletzing, F. Skiff, S. R. Bounds, and S. Vincena. Tests of collision operators using laboratory measurements of shear Alfvén wave dispersion and damping. Physics of Plasmas, 16:052110, 2009.

[27] Y. Todo, M. A. Van Zeeland, A. Bierwage, and W. W. Heidbrink. Multi-phase simulation of fast ion profile flattening due to Alfvén eigenmodes in a DIII-D experiment. Nuclear Fusion, 54:104012, 2014.

[28] T. Vernay. Numerical study of electron-ion collision effects on trapped electron modes with the gyrokinetic code ORB5. Master's thesis, École Polytechnique Fédérale de Lausanne, 2008.

[29] W. Wan, Y. Chen, and S. E. Parker. Gyrokinetic $\delta f$ simulation of the collisionless and semicollisional tearing mode instability. Physics of Plasmas, 12:012311, 2005. 